\title{
Five Ibrutinib-Associated Side Effects That All Clinicians Should Be Aware of
}

\author{
Ilana Levy ${ }^{\mathrm{a}}$ Aaron Polliack ${ }^{\mathrm{b}}$ Tamar Tadmor ${ }^{\mathrm{c}, \mathrm{d}}$ \\ ${ }^{a}$ Department of Internal Medicine B, Bnai Zion Medical Center, Haifa, Israel; ${ }^{b}$ Department of Hematology, \\ Hadassah-Hebrew University Medical Center, Jerusalem, Israel; ' ${ }^{C}$ Hematology Unit, Bnai Zion Medical Center, \\ Haifa, Israel; ${ }^{\text {d }}$ The Ruth and Bruce Rappaport Faculty of Medicine, Technion, Haifa, Israel
}

\section{Editorial comment on the paper by Miri Schamroth Pravda, Nili Schamroth Pravda, Michael Lishner. The Muddied Waters of Ibrutinib Therapy. A Case Report. Acta Haematol 2019;141:209-213}

In this issue of Acta Haematologica, Schamroth Pravda et al. [1] describe a 37-year-old male treated with ibrutinib for advanced mantle cell lymphoma who developed fatal central nervous system (CNS) aspergillosis, which was apparently not associated with any sign of diseaserelated immune suppression and probably linked to ibrutinib treatment.

Ibrutinib is an oral Bruton tyrosine kinase (BTK) inhibitor, recently approved for the treatment of mantle cell lymphoma, chronic lymphocytic leukemia (CLL), and Waldenstrom macroglobulinemia, which is also under ongoing investigation for diffuse large B-cell lymphoma and primary CNS lymphoma [2]. As the use of this effective oral compound expands, side effects are being reported, which require the treating clinicians' awareness and decisions on correct intervention. In this editorial, we have selected to describe five less known side effects of this agent which we feel are worthy of attention for everyone using ibrutinib and these are listed below.

First, and as initially described by Schamroth Pravda et al. [1], ibrutinib permits a "chemotherapy-free" approach, but bearing in mind that potentially serious infections can still be encountered. In this respect, recently reported infections include invasive fungal infections (invasive aspergillosis, Pneumocystis jirovecii pneumonia

\section{KARGER}

(c) 2019 S. Karger AG, Basel

E-Mail karger@karger.com

www.karger.com/aha
[PJP], cryptococcosis), which occur in $39 \%$ of patients with CNS lymphoma and 5\% of CLL cases treated with ibrutinib [2]. Clinical presentations may not always be conventional, and accordingly a high level of suspicion is required for diagnosis, as in the case described by Schamroth Pravda et al. [1]. In this regard, the exact underlying mechanism of this complication is still not well understood but seems to resemble the genetic BTK deficiency evident in X-linked agammaglobulinemia [1]. PJP prophylaxis and/or antifungal therapy should therefore be considered for these patients. Another infectious complication observed in ibrutinib-treated patients relates to possible hepatitis B virus (HBV) reactivation, which necessitates performing routine $\mathrm{HBV}$ status testing prior to starting therapy with ibrutinib as well as providing preventive treatment for HBV carriers [3].

Bleeding, another relatively frequent complication in patients treated with ibrutinib, occurs in about $3-5 \%$ of patients receiving the drug [4] and appears to be mediated by platelet dysfunction induced by BTK inhibition. While minor bleeding events or bruising have been more commonly described, clinicians should be aware of the risk of major bleeding, including intracranial hemorrhage which may occur in up to $2 \%$ of ibrutinib-treated patients [4]. These complications may be compounded by 
the concurrent use of anticoagulants such as vitamin $\mathrm{K}$ antagonists or other direct-acting oral anticoagulants, which may potentially interact with ibrutinib. The above data should be a "warning light" for clinicians to consider stopping ibrutinib therapy in patients who may have a high risk of falling and head trauma, as well as in those treated with anticoagulants. However, none of the above are considered absolute contraindications for ibrutinib treatment [4].

Cardiac side effects of ibrutinib mainly involve arrhythmias. The most frequently described are atrial arrhythmias in $5-8 \%$ of treated patients, and most are atrial fibrillation [4]. However, ventricular arrhythmias and sudden cardiac death have also been described in patients receiving ibrutinib [5]. The exact pathogenesis of these arrhythmias is still unclear, but clinicians must be aware of them and consider anti-arrhythmic treatment in highrisk patients, after first excluding the possibility of potential drug-drug interactions with ibrutinib.

Autoimmune side effects of ibrutinib may also occur but have been less frequently reported. One of these disorders is ibrutinib-induced autoimmune hemolytic anemia (AIHA) [6]. While AIHA may also be an autoimmune manifestation of underlying CLL (and in these cases, ibrutinib usually has a combined beneficial effect on both the CLL and the AIHA), we should remember that ibrutinib use may also be associated with AIHA exacerbation. This complication appears to be related to the lymphocytosis associated with the onset of ibrutinib therapy due to lymphocyte release from lymph nodes, causing exacerbation of autoimmune reactions such as AIHA [6]. In such cases, clinicians must realize that ibrutinib should be withheld immediately and therapy with steroids \pm rituximab administered in order to control AIHA [6].

Finally, rare nail plate abnormalities have also been reported following the initiation of ibrutinib [7]. These changes may develop within a few months of starting therapy and involve ibrutinib disruption of the disulfide bonds between cysteine residues, which are crucial for nail integrity and result in nail brittleness. Both fingernails and toenails may be involved and these alterations are generally self-limited. Lesions generally improve under weekly therapy with nail polish with a possible option of biotin treatment, without any need to stop ibrutinib [7].

In conclusion, new side effects of ibrutinib have been described since its commercial availability. While some of these may be benign (nail abnormalities), others may be threatening for the patient, particularly fungal infections, bleeding, sudden cardiac death, or unexpected exacerbation of AIHA in CLL. Clinicians using ibrutinib must obviously be aware of all these potential side effects during treatment. More research on the exact mechanisms of actions is clearly needed, and future clinical guidelines should be provided for possible prevention and routine clinical management.

\section{Disclosure Statement}

The authors have no conflicts of interest to declare. There were no funding sources for the writing of this manuscript.

\section{References}

1 Schamroth Pravda M, Schamroth Pravda N, Lishner M. The Muddied Waters of Ibrutinib Therapy. Acta Haematol. 2019. doi: 10.1159/000496555.

2 Chamilos G, Lionakis MS, Kontoyiannis DP. Call for Action: Invasive Fungal Infections Associated With Ibrutinib and Other Small Molecule Kinase Inhibitors Targeting Immune Signaling Pathways. Clin Infect Dis. 2018 Jan;66(1):140-8.
3 Hilal T, Gea-Banacloche JC, Leis JF. Chronic lymphocytic leukemia and infection risk in the era of targeted therapies: linking mechanisms with infections. Blood Rev. 2018 Sep; 32(5):387-99.

4 Boriani G, Corradini P, Cuneo A, Falanga A, Foà R, Gaidano G, et al. Practical management of ibrutinib in the real life: focus on atrial fibrillation and bleeding. Hematol Oncol. 2018 Oct;36(4):624-32.

5 Lampson BL, Yu L, Glynn RJ, Barrientos JC, Jacobsen ED, Banerji V, et al. Ventricular arrhythmias and sudden death in patients taking ibrutinib. Blood. 2017 May;129(18):2581-4.
6 Suzuki T, Miyakoshi S, Nanba A, Uchiyama T, Kawamoto K, Aoki S. A case of chronic lymphocytic leukemia complicated by autoimmune hemolytic anemia due to ibrutinib treatment. J Clin Exp Hematop. 2018 Sep; 58(3):136-40.

7 Heldt Manica LA, Cohen PR. Ibrutinib-Associated Nail Plate Abnormalities: Case Reports and Review. Drug Saf Case Rep. 2017 Nov; 4(1):15. 